\title{
Increasing incidence of hepatitis A in Bangladesh
}

\author{
M. Z. Amin ${ }^{a}$ L. N. Siddique ${ }^{b}$, M. A. Satter ${ }^{\mathfrak{c} *}$ and K. K. Biswas ${ }^{\mathrm{d}}$ \\ ${ }^{a}$ Dept. of Genetic Engineering and Biotechnology, Jessore Science and technology University, Jessore-7408, \\ Bangladesh. ${ }^{b}$ Dept of Biochemistry, Sir Salimullah Medical, Mitford, Dhaka, Bangladesh. ${ }^{c} I F S T$, BCSIR, \\ Dhaka-1205, Bangladesh and ${ }^{d}$ Dept. of Biochemistry and Molecular Biology Rajshahi University, Rajshahi, \\ Bangladesh.
}

\begin{abstract}
Hepatitis A (HAV) infection is caused by the hepatitis A virus which is transmitted through the fecal-oral route. Life long protective antibodies are present after infection. The number of cases of adult hepatitis A has progressively been increasing during the last several decades in Bangladesh. In addition, the pattern of age-specific seroprevalence of anti-HAV has changed with economic growth. The prevalence of anti-HAV in 20-40 year age range has declined rapidly during the last 3 decades. As a result, this age groups has a high risk for HAV infection and clinically overt hepatitis A is increasing in adolescents and adult. The aim of the present study were to assess whether the proportion of adults with acute HAV infection has been increasing over the years and analyze the seroprevalence of immunoglobulin M(IgM) antiHAV antibodies in young adults below the age of 20 years as well as in cases of chronic liver disease. Sera collected from 530 patients with acute and chronic liver disease attends the Somorita Hospital Ltd. during the previous 2 years and 6 months (Jan. 2008- Jun. 2010) were tested for various serological markers of acute and chronic hepatitis. In addition, 530 normal healthy attendants of the patients above the age of 20 years were tested for IgM anti-HAV as controls. Of 530 patients with acute hepatitis (13.42\%) were positive for immunoglobulin M. The patients who were IgM anti-HAV negative were found to be hepatitis B (106 patients), hepatitis C, (10 patients), hepatitis E (150 patients) and unclassified (273 patients). Although the frequency of HAV infection among young adult ( $<20$ age) had increased $(33.33 \%$ to $42.35 \%)$ in the 2 years and 6 months period, the frequency of HAV infection among adults had also increased (15.38\% to $28.13 \%)$ during the same period. This study should be helpful for the identification of high risk population for vaccination of hepatitis A.
\end{abstract}

Key Word: Hepatitis A virus, Eipdemology and Vaccination

\section{Introduction}

Hepatitis A is an enterically transmitted viral disease of global public health importance and human are known to be its principal host (Cuhbert, 2001 and Lednar et al. 1985).The ubiquitous virus is generally transmitted through the fecaloral route or through contaminated food or drink. The majority of adults develop symptoms following HAV infection but more than $70 \%$ of children younger than 6 years of age are asymptomatic or develop a mild self-limiting illness (Cotton et al. 1998). Hepatitis A infection in adults often leads to serious complications (Wilner et al. 1998 and Bell et al. 1998). Each year, approximately 100 deaths in the United States are attributed to fulminant hepatitis A infection (Craig et al. 2004).

In hyper endemic areas, most people have acquired the protective anti-HAV antibody through sub clinical childhood exposure to the virus, which confers life long immunity against HAV infections (Cotton et al. 1998). Lack of such an exposure in childhood due to control of transmission factors such as improved hygiene and easy access to basic health care support has resulted in a large non immune population in whom HAV infection has been reported to cause severe hepatitis in adults (Xu et al. 1992 and Byun et al. 2001). Several European countries have also witnessed a reduction in prevalence of HAV infection (Byun et al. 2001, Tandon et al. 1985 and Das et al. 2000). By improving hygiene, easy access to basic healthcare and vaccination.

The drop in HAV infection in young adults has resulted in the reduction of individual who have been naturally immunized and has thus increased the adult population at risk for acquiring the disease. Based on epidemiological data on the prevalence of immunoglobulin $\mathrm{G}$ (IgG) antibodies against HAV in Bangladesh, vaccination for HAV would be prevent the rate of increasing the adult population at risk for acquiring the disease.

However no comprehensive study has been conducted in Bangladesh to evaluate the shift in the HAV infection profile to indicate existence of large population of non immune sus- 
ceptible individuals, who can develop hepatitis A, if infected. Although Bangladesh is considered to be a hyper endemic region for HAV (Tandon et al. 1985). This is most significant because hepatitis A in adults pursues a protracted morbid course (Dhawan et al. 1998) and a safe and effective vaccine against this virus is now available commercially (Mall et al.2001).

More details study is needed to find out the cause of the epidemic for hepatitis A infection. So in this study we focused on the screening of the hepatitis A virus in the patients or on patient among the different age and sex groups. In this present study, we did our experiment among 530 patients during 2 years and 6 months were tested for various serological markers of acute and chronic hepatitis. The present study has therefore been designed to assess whether the proportion of adults suffering from hepatitis A has been increasing over the years. To study the seroprevalence of IgG anti-HAV antibodies in young adults above the age of 20 years and to analyze the seroprevalence of IgG anti-HAV antibody in cases of chronic liver disease.

\section{Methods}

The present study was carried out in two different groups of patients and a control group were seen during 2008 to 2010 at the Somorita Hospital Ltd., which is one of the major tertiary Hospitals in Dhaka, Bangladesh.

The first group consisted of 265 patients with acute hepatitis. All the patients were screened for the presence of immunoglobulin M (IgM) anti-HAV.

The early symptoms of the clinically diagnosed hepatitis A, which include fever, general malaise, fatigue, nausea, vomiting, anorexia and right upper quadrant discomfort.

The 2nd group consisted of 265 patients with chronic liver disease, the diagnosis of chronic liver disease was made on the basis of the conventional method such as history, clinical, biochemical and histological criteria. Patient samples were tested for the presence of HBsAg, HBV DNA, anti -HCV and HCV RNA.

In addition 530 healthy subjects above the age of less than 20 years, who accompanied patient as attendants were controlled in the study as controls.

\section{Laboratory test and Serology}

For each subject $10 \mathrm{ml}$ of venous blood was collected and separated serum samples were stored at $-20^{\circ} \mathrm{C}$ until analysis.

Patients in the first group were evaluated on the basis of history, physical examination, liver function profile and sero- logical tests for hepatitis A (ELISA, HAV).

Patients in the 2nd group (with chronic hepatitis) were screened for the presence of IgM anti-HAV using ELISA kit (Bio Rad). The patients were further evaluated on the basis of history, physical examination, liver function profile and serological tests for hepatitis $\mathrm{B}$, hepatitis $\mathrm{C}$ and hepatitis $\mathrm{E}$ using ELISA kits (Bio Rad).

Patients in the controls group were also screened for the presence of IgM-anti-HAV. Althrough the controls group had no signs of liver disease but this screened for the pre diction for the transmission of hepatitis A from the patients. The subjects were evaluated on the basis of liver function and serological tests for IgM-anti HAV assay using commercially available ELISA kit. Epidemiological information including age and sex, was elicited using a pretested questionnaire.

\section{Statistical analysis}

The difference in the number of patients with HAV-IgM, positivity during different years, and sex were tested using chi squared (x2) test of significance. The significance of age and sex were assayed by students T-test. As data revealed a positive skew with various liver function profiles, the significance of HAV-IgM positivity or negativity was analyzed by Mam-Witney U-test.

\section{Results and Discussion}

Of 265 patients diagnosed with acute hepatitis during the 2 years and 6 months period, 13.42 were HAV-IgM positive. Those who were HAV-IgM negative included hepatitis B in $(15.24 \%)$, hepatitis C in $(8.92 \%)$, hepatitis $\mathrm{E}$ in $(18.13 \%)$ and unclassified in (23.54\% ) patients (data not shown). The mean age of the HAV-IgM positive patients was 20.5. The age ranged from $0-20,21-40$ and above 40 , the male to female ratio was $1.5: 1,(340 / 190)$. The year wise distribution of HAV-IgM positive patients during 2years and 6 months study period (Jan. 2008- Jun. 2010) is shown in Table I. The

Table I: Frequency (\%) of hepatitis A (HAV- IgM) positivity during the 30 months study (Jan. 2008Jun. 2010).

\begin{tabular}{lcc}
\hline Month & Hepatitis (n) & HAV-IgM Positive[ $\mathrm{n}(\%)]$ \\
\hline Jan. 08- Jun. 08 & 95 & $08(8.42 \%)$ \\
Jul. 08- Dec. 08 & 103 & $10(9.71 \%)$ \\
Jan.09 -Jun. 09 & 106 & $12(11.32 \%)$ \\
Jul.09-Dec. 09 & 110 & $14(12.53 \%)$ \\
Jan.10-Jun.10 & 116 & $16(13.51 \%)$ \\
\hline Total & 530 & $60(13.42 \%)$ \\
\hline $\mathrm{x}^{2}=23.4 ; \mathrm{p}<0.001$ & &
\end{tabular}


overall frequency of HAV-IgM patients increased from approximately $1.35 \%$, which was found to be statistically significant $(\mathrm{p}<0.001)$.

The HAV distributions among different age groups are shown in Table II. The age of children ranged from 1 to 12 years, while young adults is ranged from 13 to 20 , (indicate in table as $<20$ years age group) adult age ranged from 21 to 40 , old aged above 40 (indicate in table as $>40$ years age group). Although the incidence of HAV infection in $<20$ years age group had increased $(10.6 \%$ to $22 \%$; $<<0.003)$, there was simultaneous increased incidence of HAV infection among adults $(<40)(3.4 \%$ to $12.3 \%$; $<<0.004)$ during the same period.

Table II: Distribution of hepatitis A(HAV- IgG) positivity during the 30 months study period (January 2008-June 210) according to age.

\begin{tabular}{lccc}
\hline Month & $<20$ & $21-40$ & $>40$ \\
\hline Jan. 08-Jun. 08 & $5(33.33 \%)$ & $6(15.38 \%)$ & $2(6.25 \%)$ \\
Jul.08-Dec. 08 & $7(41.76 \%)$ & $8(16.36 \%)$ & $4(10.31 \%)$ \\
Jan. 09-Jun. 09 & $8(42.05 \%)$ & $13(24.07 \%)$ & $5(11.36 \%)$ \\
Jul.09-Dec. 09 & $9(42.54 \%)$ & $15(24.99 \%)$ & $6(12.24 \%)$ \\
Jan. 10-Jun. 10 & 12(44.35\%) & $18(28.13 \%)$ & $8(15.39 \%)$ \\
\hline Below 20 years & \multicolumn{3}{c}{ above 20 years } \\
$x^{2}=8.7$; $<0.003$ & \multicolumn{3}{c}{$\mathrm{x}^{2}=15.3 ; \mathrm{p}<0.004$}
\end{tabular}

In 2 nd group, the age range of 265 patients with chronic liver disease was 50 years, the mean age being 37 . A total of 125 (39\%) patients were positive for HBV, HCV or dual infection or alcoholism were screened for the presence of HAVIgG antibodies and rest were of unclassified etiology hence not included in this study. Of the 265, 226(85.28\%) were positive for IgG HAV indicating sub clinical exposure to HAV infection and development of natural antibodies in these patients. With rejection to viral etiology, HBV infection was found in 207 patient of whom 163 (78.8\%) were Ig $\mathrm{M}$ anti-HAV positive, $\mathrm{HCV}$ infection was recorded in 85 , of whom 65 (76.47\%) tested positive for Ig G anti-HAV. Then patients had evidence of dual infection with $\mathrm{HBV}$ and $\mathrm{HCV}$ and all of them tested positive for IgG anti-HAV. The remaining 68 patients had past history of jaundice and alcoholism, $56(75.8 \%)$ of them had IgG anti-HAV positively.

Of healthy control subjects, who were either asymptomatic or had no clinical evidence of heptobiliary disease, 530 were enrolled using a systematic random sampling methodology by randomly selecting every third volunteer. The mean age of the subject was $32.6 \pm 13.2$ years. There were 320 men and 210 women, the ration being 1.5:1. The overall prevalence of IgG anti-HAV positivity in these subjects was $71 \%$ (376/ $530)$. In subjects older than 20 years of age $28.44 \%$ (66/232) were positive for IgM -anti-HAV, while in subject younger than 20 years, the seroprevalence was $38.59 \%(115 / 298)$ is shown in Fig.1. This finding suggests that $61.41 \%$ of the young adults in the age group $<20$ years were still negative for IgG-anti-HAV indicating susceptibility of hepatitis A infection. This trend was found to be statistically significant $\mathrm{p}<0.001$, However, there was no significant difference observed between male and female patients.

Infection with hepatitis A virus that causes viral hepatitis is endemic in many developing countries including Bangladesh. This is possibly due to lack of control of transmission factors and ubiquitous presence of HAV in the environments. In contrast, in many developed countries a significant reduction in HAV infection has been achieved because of control of possible transmission factors. However, this has led to a large population of no immune susceptible individual who can develop hepatitis A if infected.

The present study also suggests a similar trend as the number of adults, who remained uninfected had increased in due course of time. It reveals that the pattern of acute hepatitis A infection is changing, with transmission from asymptomatic childhood infection to an increased incidence of symptomatic disease in young adults ( $<20$ years). During 3 years period of study (2008 to 2010), the incidence of acute hepatitis due to HAV in young adults showed an increasing trend (shown in Figure and Table I). These results corroborate with the findings of Arankalle who reported a decrease in antiHAV positivity in children belonging to higher socio-economic categories suggesting that more hepatitis A is likely to be seen in adults (Arankalle et al.1995).

The overall prevalence of anti-HAV antibodies in patients with chronic liver disease was $47 \%$. The prevalence of antiHAV antibodies reported among the general population from various parts of Bangladesh also ranges between $85 \%-89 \%$. We did not find any significant difference between the anti-

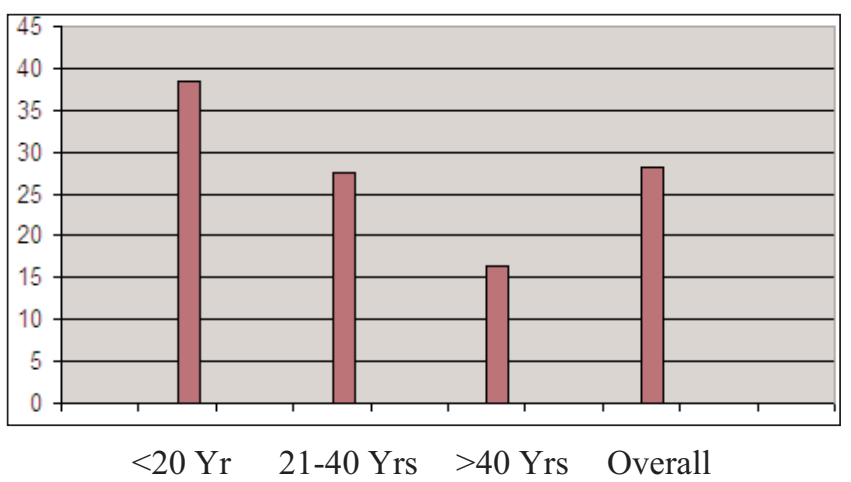

Fig. 1: Frequency (\%) of IgM anti-HAV of the participant patients according to age . 
HAV seroprevalence rate between men and women. However, they may be subgroups of patients with chronic liver disease belonging to more effluent classes who may not have had sub clinical exposure to HAV in their early childhood and in whom the subsequent development of antiHAV antibodies did not occur.

The present study was conducted with a large sample size and the appropriate diagnostic criteria for the underline disease were employed. In sharp contrast to the findings of Arankalle et al. 1995, we found 71\% positivity for anti-HAV antibodies amongst the healthy subjects as compared to $95 \%$ reported earlier. The difference is due to a rapid increase in the number of no immune individual over the last few years.

Several factors such as easier and increased access to safe drinking water, better sanitary practices and decrease fertility among women may be responsible for this epidemiological shift. The rise of infection due to changing epidemiology and the presence of a large pool of susceptible individuals will have a profound impact on the magnitude and severity of the disease. Our result suggests that hepatitis A may become a major public health problem in the country in year to come. The changes in epidemiological pattern would increase the disease burden may cause large community and lead to health care cost.

As HAV exists as a single serotype and humans are the only host, it is possible to eradicate it. In view of changing epidemiology of HAV infection in Bangladesh and the increase incidence of acute hepatitis A amongst the young adults as evident from this study, it is suggested that in Bangladesh, any individual above the age of 10 years who is seronegative for IgM -anti-HAV should be vaccinated against hepatitis A to reduce the risk of contacting the infection in adult life.

\section{References}

Arankalle VA, Tsarev, SA and Chandha MS (1982 \& 1995). Age specific prevalence antibodies to hepatitis A and E virus in pune, India. J. Infect. Dis.171: 447-450.

Bell BP, Shapiro VN and Alter MJ (1998). The diverse patterns of hepatitis A epidemiology in the United States: implications for vaccination strategies. J. Infect. Dis. 178: 1579-1584.

Byun KS, Kim JH and Song KG (2001). Molecular epidemiology of the hepatitis A virus in korea. $J$. Gastroenterol. Hepatol.16: 519-24.
Cotton MG and Locarnini SA (1998). Hepatitis A virus epidemiology. In: Zuckermann AJ, Thomas HC, eds.Viral hepatitis. London: Churchill Livingstone. 29-41.

Cuhbert JA (2001). Hepatitis A: old and new. Clin. Microbiol. Rev.14: 35-58.

Craig AS and Schaffner W(2004). Prevention of hepatitis A with the hepatitis A vaccine. N. Engl. J. Med.350: 476481 .

Das K, Jain A and Gupta (2000). The changing epidemiological pattern of hepatits A virus in an urban population in India: emergence of a trend similar to European countries. Eur. J. Epidemiol. 16: 507-510.

Dhawan PS, Shah SS and Alvareas JF (1998). Seroprevalence of hepatitis A in Mumbai and immunogenicity and safety of hepatitis A vaccine. Indian $J$. Gastroenterol.17: 16-18.

Lednar WM, Lemon SM, Kirkpatrick JW, Redfield RR, Fields ML and Kelly PW (1985). Frequency of illness associated with epidemic hepatitis A virus infection in adults. Am. J. Epidemiol.122: 226-33.

Mall ML, Rai RR and Phillip M (2001). Seroepidemiology of hepatitis A in India: Changing pattern. Indian. $J$. Gastroenterol. 20: 130-132.

Tanaka J (2000). Hepatitis A shifting epidemiology in Latin America. Vaccine.18 (Suppl.1): S57-60.

Tandon BN, Gandhi BM, Joshi YK, Irshad M and Gupta H (1985). Hepatitis virus non -A, non B,: The cause of a major public problem in India. Bull. World Health Organ. 63: 931-934.

Wilner IR, Uhl MD, Howard SC, Williams EQ, Riely CA and Waters B (1998). Serous hepatitis A: an analysis of patients hospitalized during an urban epidemic in the United States. Ann. Intern. Med.128: 111-114.

Xu ZY, Li ZH, Wang JX, Xiao ZP and Dong DX (1992). Etiology and prevenation of a shellfish associated epidemic in Shanghai, China. Vaccine. 10 (Suppl.): $367-$ 380 .

Received : 26 July 2011; Revised : 20 September 2011, Accepted : 13 October 2011. 